\title{
NON-OSCILLATORY CENTRAL SCHEMES FOR THE INCOMPRESSIBLE 2-D EULER EQUATIONS
}

\author{
Doron LEVy AND EITAN TADMOR
}

\begin{abstract}
We adopt a non-oscillatory central scheme, first presented in the context of Hyperbolic conservation laws in [28] followed by [15], to the framework of the incompressible Euler equations in their vorticity formulation. The embedded duality in these equations, enables us to toggle between their two equivalent representations - the conservative Hyperbolic-like form vs. the convective form. We are therefore able to apply local methods, to problems with a global nature. This results in a new stable and convergent method which enjoys high-resolution without the formation of spurious oscillations. These desirable properties are clearly visible in the numerical simulations we present.
\end{abstract}

\section{Introduction}

We are concerned with the approximate solution of fluid flows governed by the following system of Euler equations,

$$
\vec{u}_{t}+(\vec{u} \cdot \nabla) \vec{u}=-\nabla p,
$$

which is augmented with the incompressibility constraint, $\nabla \cdot \vec{u}=0$, and is subject to initial conditions, $\vec{u}(\vec{x}, 0)=\vec{u}_{0}(\vec{x})$. Here, $\vec{u}$ and $p$ denote, respectively, the velocity field and the pressure.

In two space dimensions, system (1.1) admits an equivalent scalar formulation in terms of the vorticity, $\omega:=\nabla \times \vec{u}$, which satisfies the conservative scalar equation,

$$
\omega_{t}+(u \omega)_{x}+(v \omega)_{y}=0
$$

Here, $\vec{u}=(u, v)$, is the two-component divergence-free velocity field, satisfying

$$
u_{x}+v_{y}=0
$$

Received May 3, 1996.

1991 Mathematics Subject Classification: Primary 65M10; Secondary 76C05.

Key words and phrases: Hyperbolic conservation laws, second-order accuracy, central difference schemes, non-oscillatory, schemes, incompresible Euler equations.

Research was supported by DARPA/ONR Grant \#N00014-92-J-1890, ONR Grant \#N00014-91-J-1076, NSF Grant \#DMS94-04942 and by the Sackler Institute for Scientific Computations in TAU. Part of the work of the first author was carried out at the Mathematics Department of UCLA, and he would also like to thank the department for its warm hospitality. 
Equation (1.2) can be viewed as a nonlinear conservation law,

$$
\omega_{t}+f(\omega)_{x}+g(\omega)_{y}=0
$$

with a global flux, $(f, g):=(u \omega, v \omega)$. At the same time, the incompressibility (1.3) enables us to rewrite (1.2) in the equivalent convective form

$$
\omega_{t}+u \omega_{x}+v \omega_{y}=0 .
$$

Equation (1.5) guarantees that the vorticity, $\omega$, propagates with finite speed, at least for uniformly bounded velocity field, $\vec{u} \in L^{\infty}$. This duality - between the conservative and convective forms of the equations, plays an essential role in our discussion below.

In recent years, there was an enormous amount of successful activity in the construction, analysis and implementation of modern numerical algorithms for the approximate solution of nonlinear hyperbolic conservation laws (1.4). A large variety of accurate, high-resolution methods were developed and investigated, e.g. [21], [10], and the references therein. We are therefore motivated to borrow the methods and ideas developed in this context. Godunov-type schemes are primary examples for these modern high-resolution schemes. Such schemes are based on piecewise-polynomial reconstruction of pointvalues from cell averages, followed by the evolution of approximate fluxes. We distinguish between upwind and central Godunov-type schemes. The difference between these two types, lies in the way they realize the evolution of these piecewise-polynomials: Upwind schemes sample the reconstructed values at the midcells. They necessitate characteristic information (approximate Riemann solvers...) and dimensional splitting, consult [13],[19] and [31], for example. Central schemes are based on staggered sampling at the interfacing breakpoints. Their main advantage is simplicity, consult [9],[28] and [15].

To be more specific, we concentrate on multidimensional extensions of the non-oscillatory, second-order central Nessyahu-Tadmor (NT) scheme [28]. The central framework starts, at each time-level, with a non-oscillatory piecewise linear approximation which is reconstructed from the piecewise constant numerical data. This piecewise-linear approximation is evolved to the next time level and then realized by its piecewise constant projection. The projection is based on staggered averaging which covers both left going and right going waves centered at each midcell. Consequently, the evolution step utilizes smooth numerical fluxes, which are bounded away from the center of the discontinuous Riemann fans. And here, approximate quadrature rules can replace the costly (approximate) Riemann solvers embedded in upwind schemes. It is therefore natural to use this central framework in more than one space dimension - where we avoid Riemann solvers and dimensional splitting. In this context we refer to the two-dimensional central scheme recently introduced by Jiang and Tadmor [15].

The paper is organized as follows. In $\S 2$ we briefly overview the central framework, including the two-dimensional central-scheme [15]; we also outline a new two-dimensional third-order extension along the lines of Liu and Tadmor in 
the one dimensional case [26]. In $\S 3$ we utilize this central framework, introducing our central approximation of the incompressible Euler equations (1.2)(1.3). We note in passing that a similar treatment applies to the incompressible Navier-Stokes equations, where the central discretization of its convective terms is complemented with an implicit Crack-Nicholson discretization of the additional parabolic terms.

In $\S 4$, we carry out stability analysis, which proves that our two-dimensional second-order central scheme satisfies the scalar maximum principle (for the vorticity). This, in turn, implies by compensated compactness arguments, that there is no concentration effect [8], and hence the convergence of our central scheme follows, at least for $\omega_{0} \in L^{p}, p>2$, [22]. In $\S 5$ we briefly remark on the boundary treatment for our central scheme. For the intricate issue of the recovery of the vorticity boundary values from the velocity field we refer to [25]. Given the vorticity boundary values, we may then utilize the boundary treatment presented in the general Hyperbolic context [23]. Most importantly, we present here a general velocity reconstruction that retains the discrete incompressibility relation required by the maximum principle in $\S 4$; unlike the velocity reconstruction in $\S 3$, it is not limited to the periodic case.

We end up in $\S 6$, with a couple of prototype numerical examples. We present the problem of an incompressible jet in a doubly periodic geometry subject to two different sets of initial parameters. First, following Bell, Colella and Glaz [3], we consider the case of the so-called "thick" shear-layer: the numerical simulations obtained for this problem demonstrate the stability and convergence properties of our central schemes. Second, following Brown and Minion [4], we then proceed with a framework which involves smaller scales, the so-called "thin" shear-layer. Here, our central scheme resolves the incompressible solution with no spurious vortices, which are inherent with other numerical methods reported in the literature, e.g., [4],[32]. Our numerical experiments show a remarkable speedup while retaining stability and high-resolution.

\section{The two-dimensional central scheme - a brief overview}

We start this section with a brief review of the central framework presented in [15]. This will enable us to introduce the methodology and notations to be used later. We consider the two-dimensional hyperbolic system of conservation laws

$$
u_{t}+f(u)_{x}+g(u)_{y}=0
$$

subject to the initial data, $u(x, y, t=0)=u_{0}(x, y)$. To approximate (2.1) by a central scheme, we introduce a piecewise-polynomial approximate solution, $w(\cdot, \cdot, t)$, at the discrete time levels, $t^{n}=n \Delta t$,

$$
w\left(x, y, t^{n}\right)=\sum_{j, k} p_{j, k}(x, y) \chi_{j, k}(x, y), \quad \chi_{j, k}(x, y):=1_{I_{j, k}},
$$


where $p_{j, k}(x, y)$ are polynomials supported at the cells,

$$
I_{j, k}:=\left\{(\xi, \zeta)|| \xi-x_{j}\left|\leq \frac{\Delta x}{2},\right| \zeta-y_{k} \mid \leq \frac{\Delta y}{2}\right\} .
$$

An exact evolution of $w$, based on integration of the conservation law (2.1) over the staggered control volume, $I_{j+\frac{1}{2}, k+\frac{1}{2}} \times\left[t^{n}, t^{n+1}\right]$, yields

$$
\begin{aligned}
& \bar{w}_{j+\frac{1}{2}, k+\frac{1}{2}}^{n+1}=\frac{1}{\Delta x \Delta y} \iint_{I_{j+\frac{1}{2}, k+\frac{1}{2}}} w\left(x, y, t^{n}\right) d y d x \\
& -\frac{1}{\Delta x \Delta y} \int_{\tau=t^{n}}^{t^{n+1}}\left\{\int_{y=y_{k}}^{y_{k+1}}\left[f\left(w\left(x_{j+1}, y, \tau\right)\right)-f\left(w\left(x_{j}, y, \tau\right)\right)\right] d y\right\} d \tau \\
& -\frac{1}{\Delta x \Delta y} \int_{\tau=t^{n}}^{t^{n+1}}\left\{\int_{x=x_{j}}^{x_{j+1}}\left[g\left(w\left(x, y_{k+1}, \tau\right)\right)-g\left(w\left(x, y_{k}, \tau\right)\right)\right] d x\right\} d \tau .
\end{aligned}
$$

Here, $\bar{w}_{i l}^{n}$, is the cell average at $t=t^{n}$ associated with the cell $I_{i l}$. Thus, the first integral on the RHS represents the staggered cell average at time $t^{n}, \bar{w}_{j+\frac{1}{2}, k+\frac{1}{2}}^{n}$. It consists of contributions from the four neighboring cells,

$$
\begin{aligned}
& \bar{w}_{j+\frac{1}{2}, k+\frac{1}{2}}^{n}:=\frac{1}{\Delta x \Delta y} \iint_{I_{j+\frac{1}{2}, k+\frac{1}{2}}} w\left(x, y, t^{n}\right) d y d x= \\
& \frac{1}{\Delta x \Delta y}\left[\int_{x_{j}}^{x_{j+\frac{1}{2}}} \int_{y_{k}}^{y_{k+\frac{1}{2}}} p_{j, k}(x, y, t) d y d x+\int_{x_{j}}^{x_{j+\frac{1}{2}}} \int_{y_{k}+\frac{1}{2}}^{y_{k+1}} p_{j, k+1}(x, y, t) d y d x\right. \\
& \left.\quad+\int_{x_{j+\frac{1}{2}}}^{x_{j+1}} \int_{y_{k}}^{y_{k+\frac{1}{2}}} p_{j+1, k}(x, y, t) d y d x+\int_{x_{j+\frac{1}{2}}}^{x_{j+1}} \int_{y_{k+\frac{1}{2}}}^{y_{k+1}} p_{j+1, k+1}(x, y, t) d y d x\right] .
\end{aligned}
$$

These integrals can be evaluated exactly. It remains to recover the pointvalues $\left\{w(\cdot, \cdot, \tau) \mid t^{n} \leq \tau \leq t^{n+1}\right\}$, a task which is accomplished in two steps. First, we use the given cell averages to reconstruct the pointvalues of $w\left(\cdot, \cdot, t^{n}\right)$, reconstructed as piecewise polynomial approximation. Second, we follow the evolution of these pointvalues along the interfaces $\left(x_{j}, y_{k}, \tau\right), t^{n} \leq \tau \leq t^{n+1}$. It is here that we take advantage of the finite speed of propagation, guaranteed by the convective form (1.5): Thanks to staggering, these interfaces remain free of discontinuities, at least for a sufficiently small time step, $\Delta t$, dictated by the CFL constraint. Hence, the numerical fluxes - which remain bounded away from the propagating singularity at $\left(x_{j+\frac{1}{2}, k+\frac{1}{2}}\right)$, can be computed within any degree of desired accuracy by appropriate quadrature rules.

Below, we present two possible constructions of such central schemes - the second-order by Jiang and Tadmor, [15], which utilizes the MUSCL piecewise linear interpolant [19]; In addition we introduce a third-order two-dimensional extension of the one-dimensional central scheme by Liu and Tadmor, [26], which utilizes the non-oscillatory piecewise-parabolic interpolant from [24]. 
2.1. The second-order central NT scheme. Following the two-dimensional scheme in [15], which extends the one-dimensional NT scheme in [28], we start with a reconstructed piecewise-linear MUSCL approximation,

$$
w\left(x, y, t^{n}\right)=\sum_{j, k} p_{j, k}(x, y) \chi_{j, k}(x, y)
$$

where,

$$
p_{j, k}(x, y)=\bar{w}_{j, k}^{n}+w_{j, k}^{\prime}\left(\frac{x-x_{j}}{\Delta x}\right)+w_{j, k}^{\prime}\left(\frac{y-y_{k}}{\Delta y}\right) .
$$

Here, $w_{j, k}^{\prime}$ and $w_{j, k}^{\prime}$, are respectively, the discrete slopes in the x-direction and in the y-direction, which are reconstructed from the given cell averages. Second order accuracy is guaranteed wherever these slopes approximate the corresponding derivatives, $w_{j, k}^{\prime} \sim \Delta x \cdot w_{x}\left(x_{j}, y_{k}, t^{n}\right)+O(\Delta x)^{2}, w_{j, k}^{\prime} \sim \Delta y \cdot w_{y}\left(x_{j}, y_{k}, t^{n}\right)+$ $O(\Delta y)^{2}$. With this choice of linear approximation, the first term on the RHS of $(2.2)$ - the staggered average, $\bar{w}_{j+\frac{1}{2}, k+\frac{1}{2}}^{n}$, yields by a straightforward computation,

$$
\begin{gathered}
\bar{w}_{j+\frac{1}{2}, k+\frac{1}{2}}^{n}=\frac{1}{4}\left(\bar{w}_{j, k}^{n}+\bar{w}_{j, k+1}^{n}+\bar{w}_{j+1, k}^{n}+\bar{w}_{j+1, k+1}^{n}\right) \\
+\frac{1}{16}\left(w_{j, k}^{\prime}-w_{j+1, k}^{\prime}+w_{j, k+1}^{\prime}-w_{j+1, k+1}^{\prime}\right) \\
+\frac{1}{16}\left(w_{j, k}^{\prime}-w_{j, k+1}^{\prime}+w_{j+1, k}^{\prime}-w_{j+1, k+1}^{\prime}\right) .
\end{gathered}
$$

Next, we turn to the numerical fluxes on the RHS of equation (2.2). They are approximated by the second-order midpoint quadrature rule for the time integral, and by the second-order rectangular quadrature rule for the spatial integration. For example, approximation of the first flux on the right yields

$$
\int_{\tau=t^{n}}^{t^{n+1}} \int_{y=y_{k}}^{y_{k+1}} f\left(w\left(x_{j+1}, y, \tau\right)\right) d y d \tau \sim \frac{\Delta t \Delta y}{2}\left(f_{j+1, k}^{n+\frac{1}{2}}+f_{j+1, k+1}^{n+\frac{1}{2}}\right) .
$$

Analogous expressions hold for the remaining fluxes. The missing midvalues, $w_{j, k}^{n+\frac{1}{2}}$, are predicted using a first-order Taylor expansion (where $\lambda:=\frac{\Delta t}{\Delta x}$ and $\mu:=\frac{\Delta t}{\Delta y}$, are the usual fixed mesh-ratios),

$$
w_{j, k}^{n+\frac{1}{2}}=w_{j, k}^{n}-\frac{\lambda}{2} f_{j, k}^{\prime}-\frac{\mu}{2} g_{j, k}^{\prime} .
$$

Equipped with these midvalues, we are now able to use the approximate fluxes outlined in (2.4), which yield a second-order corrector step of the form

$$
\begin{aligned}
\bar{w}_{j+\frac{1}{2}, k+\frac{1}{2}}^{n+1}= & <\frac{1}{4}\left(\bar{w}_{j, .}^{n}+\bar{w}_{j+1, .}^{n}\right)+\frac{1}{8}\left(w_{j, .}^{\prime}-w_{j+1, .}^{\prime}\right)-\lambda\left(f_{j+1, .}^{n+\frac{1}{2}}-f_{j, .}^{n+\frac{1}{2}}\right)>_{k+\frac{1}{2}} \\
(2.6)+ & <\frac{1}{4}\left(\bar{w}_{., k}^{n}+\bar{w}_{., k+1}^{n}\right)+\frac{1}{8}\left(w_{., k}^{\prime}-w_{., k+1}^{\prime}\right)-\mu\left(g_{., k+1}^{n+\frac{1}{2}}-g_{., k}^{n+\frac{1}{2}}\right)>_{j+\frac{1}{2}} .
\end{aligned}
$$


Here, we employ the following abbreviation for staggered-averaging

$$
<w_{j, .}>_{k+\frac{1}{2}}:=\frac{1}{2}\left(w_{j, k}+w_{j, k+1}\right), \quad<w_{., k}>_{j+\frac{1}{2}}:=\frac{1}{2}\left(w_{j, k}+w_{j+1, k}\right) .
$$

Note that the predictor-corrector central scheme, (2.5)-(2.6), is an extension to the canonical first-order Lax-Friedrichs scheme based on piecewise-constant reconstruction, (with $p_{j, k} \equiv \bar{w}_{j, k}$ and $w_{j, k}^{\prime}=w_{j, k}^{\prime}=0$ ). It is remarkable that such a relatively simple extension yields a considerable improvement in the resolution of the first-order Lax-Friedrichs scheme, while retaining its robust stability properties.

2.2. A third-order extension. We extend the work of Liu and Tadmor [26] who dealt with a third-order one-dimensional central scheme. To extend it for the two-dimensional framework, we start with a piecewise parabolic reconstruction, $w\left(x, y, t^{n}\right)=\sum_{j, k} p_{j, k}(x, y) \chi_{j, k}(x, y)$, which consists of quadratic pieces of the form (ignoring mixed terms)

$$
\begin{aligned}
p_{j, k}(x, y)= & w_{j, k}^{n}+w_{j, k}^{\prime}\left(\frac{x-x_{j}}{\Delta x}\right)+\frac{1}{2} w_{j, k}^{\prime \prime}\left(\frac{x-x_{j}}{\Delta x}\right)^{2} \\
& +w_{j, k}^{\prime}\left(\frac{y-y_{k}}{\Delta y}\right)+\frac{1}{2} w_{j, k}^{\prime \prime}\left(\frac{y-y_{k}}{\Delta y}\right)^{2} .
\end{aligned}
$$

The conservation requires that the cell average of $p_{j, k}(x, y)$ coincide with the underlying given average $\bar{w}_{j, k}$, i.e., we require $\bar{p}_{j, k}=\bar{w}_{j, k}$; in addition, we place the further constraints that the cell averages of $p_{j, k}$ over the four neighboring cells coincide with their underlying given averages, $\bar{w}_{j \pm 1, k \pm 1}$. By that, the free five coefficients in (2.7) are uniquely determined as follows. We start with the reconstructed pointvalues, $w_{j, k}^{n}$; unlike the second-order schemes, these pointvalues need not coincide with the cell averages, and are given by

$$
w_{j, k}^{n}:=\bar{w}_{j, k}-\frac{1}{24} w_{j, k}^{\prime \prime}-\frac{1}{24} w_{j, k}^{\prime \prime} .
$$

Next, the first-order discrete slopes, $w_{j, k}^{\prime}$ and $w_{j, k}^{\prime}$, are reconstructed as follows ${ }^{1}$,

$$
w_{j, k}^{\prime}:=\theta_{j, k} \Delta_{0}^{x} \bar{w}_{j, k}^{n}, \quad w_{j, k}^{\prime}:=\theta_{j, k} \Delta_{0}^{y} \bar{w}_{j, k}^{n},
$$

and finally, double-primes stands for the reconstructed discrete second derivatives

$$
w_{j, k}^{\prime \prime}:=\theta_{j, k} \Delta_{+}^{x} \Delta_{-}^{x} \bar{w}_{j, k}, \quad w_{j, k}^{\prime \prime}:=\theta_{j, k} \Delta_{+}^{y} \Delta_{-}^{y} \bar{w}_{j, k} .
$$

The extra free parameters, $\theta_{j, k},\left(0<\theta_{j, k} \leq 1\right)$, are limiters designed to avoid spurious extrema, so that they guarantee the overall non-oscillatory nature of the central scheme. Generically, $\theta_{j, k}=1-O\left((\Delta x)^{3}+(\Delta y)^{3}\right)$, retains the third-order accuracy in most of the computational domain, with the possible exception at

\footnotetext{
${ }^{1}$ Here and below, we used the usual notations for the one-sided and centered differences, i.e., $\Delta_{ \pm} w(x)= \pm(w(x \pm \Delta x)-w(x))$ and $\Delta_{0}=\frac{1}{2}\left(\Delta_{+}-\Delta_{-}\right)$.
} 
critical cells. For further details on the reconstruction of such one-dimensional limiters consult, e.g., [24],[26].

The staggered averages on the RHS of (2.2) yield the same formula as in the second-order scheme, consult (2.4). As with the second-order scheme, the piecewise-parabolic reconstruction (2.7), is also evolved in time using the central Godunov-type framework. To retain third-order accuracy, however, we use the Simpson (rather than the midpoint) quadrature rule for time integration.

To this end, we first use the Taylor expansion to predict the midvalues, $w_{j, k}^{n+\frac{1}{2}}$ and $w_{j, k}^{n+1}$,

$$
\begin{aligned}
& w_{j, k}^{n+\frac{1}{2}}=w_{j, k}^{n}+\left(\frac{\Delta t}{2}\right) \dot{w}_{j, k}^{n}+\frac{(\Delta t)^{2}}{8} \ddot{w}_{j, k}^{n}, \\
& w_{j, k}^{n+1}=w_{j, k}^{n}+\Delta t \dot{w}_{j, k}^{n}+\frac{(\Delta t)^{2}}{2} \ddot{w}_{j, k}^{n} .
\end{aligned}
$$

Here, $\dot{w}_{j, k}^{n}$ and $\ddot{w}_{j, k}^{n}$, denote, respectively, the first and second time derivatives, which are replaced by spatial discrete derivatives as told by the conservation law (2.1).

These predicted values are then used in conjunction with the Simpson rule, yielding the corrector step

$$
\begin{aligned}
\bar{w}_{j+\frac{1}{2}, k+\frac{1}{2}}^{n+1}= & <\frac{1}{4}\left(\bar{w}_{j, .}^{n}+\bar{w}_{j+1, .}^{n}\right)+\frac{1}{8}\left(w_{j, .}^{\prime}-w_{j+1, .}^{\prime}\right)>_{k+\frac{1}{2}} \\
& +<\frac{1}{4}\left(\bar{w}_{., k}^{n}+\bar{w}_{., k+1}^{n}\right)+\frac{1}{8}\left(w_{., k}^{\prime}-w_{., k+1}^{\prime}\right)>_{j+\frac{1}{2}}
\end{aligned}
$$

$-\frac{\lambda}{6}\left[<f_{j+1, .}^{n}-f_{j, .}^{n}>_{k+\frac{1}{2}}+4<f_{j+1, .}^{n+\frac{1}{2}}-f_{j, .}^{n+\frac{1}{2}}>_{k+\frac{1}{2}}+<f_{j+1, .}^{n+1}-f_{j, .}^{n+1}>_{k+\frac{1}{2}}\right]$

$-\frac{\mu}{6}\left[<g_{., k+1}^{n}-g_{., k}^{n}>_{j+\frac{1}{2}}+4<g_{., k+1}^{n+\frac{1}{2}}-g_{., k}^{n+\frac{1}{2}}>_{j+\frac{1}{2}}+<g_{., k+1}^{n+1}-g_{., k}^{n+1}>_{j+\frac{1}{2}}\right]$.

\section{The central incompressible scheme}

We now turn our attention to the two-dimensional incompressible Euler equations, (1.2), which we view as a two-dimensional nonlinear conservation law with flux, $(f, g)=(u \omega, v \omega)$. We are aware, of course, that this is not an Hyperbolic equation, due to the global dependence of the flux on $\omega$, which can be read from the Biot-Savart law,

$$
\vec{u}(\vec{x}, t)=\int \vec{K}\left(\vec{x}-\overrightarrow{x^{\prime}}\right) \omega\left(\overrightarrow{x^{\prime}}, t\right) d \overrightarrow{x^{\prime}}, \quad \vec{K}(\vec{x}):=\frac{(-y, x)}{2 \pi|\vec{x}|^{2}} .
$$

Yet, according to the convective form (1.5), the vorticity, $\omega$, propagates with a finite speed, as long as the velocities, $u, v$, remain uniformly bounded. This convective formulation (due to the incompressibility), is the key property which enables us to utilize the central schemes (2.5)-(2.6), (2.11)-(2.12) - schemes which are of inherent "local" nature, in this context of "global" incompressible equations. 
In every step of the incompressible computation, one has to reconstruct the velocity field, $\vec{u}$, from the known values of the vorticity, $\omega\left(\cdot, \cdot, t^{n}\right)$, according to the Biot-Savart law (3.1). This could be implemented in one of several ways, consult e.g., [2],[3],[4],[6],[7],[14],[32]. We shall mention two options.

For a periodic setup, for example, this reconstruction can be done efficiently using spectral methods. Thus, by applying the Fourier transform for the elliptic system

$$
u_{x}+v_{y}=0, \quad v_{x}-u_{y}=\omega
$$

we obtain

$$
\hat{v}(\vec{k})=-\frac{\imath k_{x}}{k_{x}^{2}+k_{y}^{2}} \hat{\omega}(\vec{k}), \hat{u}(\vec{k})=\frac{\imath k_{y}}{k_{x}^{2}+k_{y}^{2}} \hat{\omega}(\vec{k}), \hat{u}(\vec{k})=\frac{1}{2 \pi} \int_{\vec{x}} u(\vec{x}) e^{-\imath \vec{k} \cdot \vec{x}} d \vec{x} .
$$

Alternatively, we can use a streamfunction, $\psi$, such that $\Delta \psi=-\omega$, which is obtained, e.g., by solving the five-points Laplacian, $\Delta \psi_{j, k}=-\omega_{j, k}$. Then, its gradient, $\nabla \psi$ recovers the velocity field

$$
u_{j, k+\frac{1}{2}}=\frac{\psi_{j, k+1}-\psi_{j, k}}{\Delta y}, \quad v_{j+\frac{1}{2}, k}=\frac{-\psi_{j+1, k}+\psi_{j, k}}{\Delta x} .
$$

Observe that in this way, we retain the discrete incompressibility, centered around $\left(j+\frac{1}{2}, k+\frac{1}{2}\right)$,

$$
\Delta_{+}^{x} u_{j, k+\frac{1}{2}}+\Delta_{+}^{y} v_{j+\frac{1}{2}, k}=0 .
$$

To define the velocity field at the integer gridpoints, $\left(x_{j}, y_{k}\right)$, required in the predictor steps (2.5) and (2.11), we may now solve

$$
\frac{1}{2}\left(u_{j, k+1}+u_{j, k}\right):=u_{j, k+\frac{1}{2}}, \quad \frac{1}{2}\left(v_{j, k}+v_{j+1, k}\right):=v_{j+\frac{1}{2}, k} .
$$

Observe that with this integer indexed velocity field, the discrete incompressibility relation (3.5) amounts to

$$
\frac{<u_{j+1, \cdot}-u_{j, \cdot}>_{k+\frac{1}{2}}}{\Delta x}+\frac{<v_{\cdot, k+1}-v_{\cdot, k}>_{j+\frac{1}{2}}}{\Delta y}=0 .
$$

The discrete incompressibility relation (3.7) will enable us to reformulate our central scheme (2.6), in an equivalent convective form, which, in turn, is responsible for a maximum principle proved in $\S 4$. We should emphasize that different schemes require different discrete incompressibility relations in order to guarantee consistency with both the conservative and the convective form of the vorticity equation, (1.2) and (1.5). A different discrete incompressibility relation in the context of upwind schemes was originally introduced in [25].

We are ready to introduce our central approximation of the two-dimensional equations (1.2)-(1.3). Assume the cell-averages of the vorticity at time $t=t^{n}$, 
$\bar{\omega}_{j, k}^{n}$, are known. Then the following algorithm calculates the staggered cellaverages of the vorticity, $\bar{\omega}_{j+\frac{1}{2}, k+\frac{1}{2}}^{n+1}$, at the next time step, $t=t^{n+1}$.

\section{Algorithm:}

\section{Reconstruction}

(1a) Reconstruct the discrete vorticity slopes.

For example, for the second-order method, calculate $\omega_{j, k}^{\prime}$ and $\omega_{j, k}^{\prime}$, with the following family of so-called Min-Mod limiters, see e.g., [13],[33].

$$
\begin{aligned}
& \omega_{j, k}^{\prime}=M M\left\{\theta\left(\bar{\omega}_{j+1, k}^{n}-\bar{\omega}_{j, k}^{n}\right), \frac{1}{2}\left(\bar{\omega}_{j+1, k}^{n}-\bar{\omega}_{j-1, k}^{n}\right), \theta\left(\bar{\omega}_{j, k}^{n}-\bar{\omega}_{j-1, k}^{n}\right)\right\}, \\
& \omega_{j, k}^{\prime}=M M\left\{\theta\left(\bar{\omega}_{j, k+1}^{n}-\bar{\omega}_{j, k}^{n}\right), \frac{1}{2}\left(\bar{\omega}_{j, k+1}^{n}-\bar{\omega}_{j, k-1}^{n}\right), \theta\left(\bar{\omega}_{j, k}^{n}-\bar{\omega}_{j, k-1}^{n}\right)\right\} .
\end{aligned}
$$

Here, $M M$, denotes the Min-Mod (MM) function,

$$
M M\left\{x_{1}, x_{2}, \ldots\right\}= \begin{cases}\min _{i}\left\{x_{i}\right\} & \text { if } x_{i}>0, \forall i \\ \max _{i}\left\{x_{i}\right\} & \text { if } x_{i}<0, \forall i \\ 0 & \text { otherwise }\end{cases}
$$

and $\theta, 0<\theta<2$, is a free parameter, which retains the non-oscillatory properties of the approximate solution. For the third-order method, the first and the second-order discrete slopes are outlined in (2.9)-(2.10).

(1b) Calculate the pointvalues of the vorticity, $\omega_{j, k}^{n}$, at time $t=t^{n}$.

Note that in the first-order and second-order approximations, these pointvalues coincide with the given cell averages, $\omega_{j, k}^{n}=\bar{\omega}_{j, k}^{n}$. Starting with the third order method, however, pointvalues may differ from the cell averages. For example, by (2.8), the third-order accurate pointvalues are given by

$$
\omega_{j, k}^{n}=\bar{\omega}_{j, k}-\frac{1}{24} \omega_{j, k}^{\prime \prime}-\frac{1}{24} \omega_{j, k}^{\prime \prime}
$$

\section{Prediction}

(2a) Prepare the pointvalues of the divergence-free velocity field, $\vec{u}\left(\cdot, \cdot, t^{n}\right)$, from the reconstructed vorticity pointvalues, $w_{j, k}^{n}$. To this end, use a direct

summation of the Biot-Savart relation (3.1), or any of its equivalent procedures mentioned earlier - spectral (3.3), streamfunction solver (3.4)-(3.6),...

(2b) Predict the midvalues of the vorticity, $\omega_{j, k}^{n+\frac{1}{2}}$.

For example, in the second-order case we use

$$
\omega_{j, k}^{n+\frac{1}{2}}=\omega_{j, k}^{n}-\frac{\lambda}{2} u^{n} \omega_{j, k}^{\prime}-\frac{\mu}{2} v_{j, k}^{n} \omega_{j, k}^{\prime} .
$$

Observe that here we use the predictor step (2.5) in its convective formulation $(1.5)$, that is, $\left(f^{\prime}, g^{\prime}\right)=\left(u \omega^{\prime}, v \omega^{\prime}\right)$. For the third order scheme, we also have to predict the pointvalues of the vorticity at time $t^{n+1}$ as well, utilizing (2.11). 


\section{Correction}

(3a) As in step (2a), use the previously calculated values of the vorticity to compute the divergence-free pointvalues of the velocity, at time $t^{n+\frac{1}{2}}$, $\vec{u}\left(\cdot, \cdot, t^{n+\frac{1}{2}}\right),\left(-\right.$ and at time $t^{n+1}$ for the third-order method).

(3b) Finally, the previously calculated pointvalues of the velocities and vorticity are plugged into the second-order corrector step (2.6) (- or (2.12) in the thirdorder method), to compute the staggered cell-averages of the vorticity at time $t^{n+1}, \bar{\omega}_{j+\frac{1}{2}, k+\frac{1}{2}}^{n+1}$.

We close this section by noting that this algorithm which deals only with the convective terms, can be extended to handle parabolic terms. As a direct consequence, the central schemes presented above, can be applied to the two-dimensional incompressible Navier-Stokes equations, $\omega_{t}+(u \omega)_{x}+(v \omega)_{y}=$ $\frac{1}{\operatorname{Re}}\left(\omega_{x x}+\omega_{y y}\right)$, with $u_{x}+v_{y}=0$. In terms of stability considerations, the usage of the implicit Crack-Nicholson scheme for handling the parabolic terms, is preferable.

\section{The maximum principle}

In this section we prove that under appropriate CFL condition, our secondorder central scheme satisfies a maximum principle. The approximate solution therefore imitates the maximum principle of the exact vorticity solution.

The theorem we state and prove, is similar to that of Jiang and Tadmor [15], in the context of scalar conservation laws. However, this equivalence is far from being trivial due to the global nature of our non-local "fluxes". In order to apply the methods of [15] in our context, it is essential to take advantage of an appropriate discrete formulation of the incompressibility condition.

In the following, we let $U_{\infty}:=\max _{j, k}\left\{\left|u_{j, k}\right|,\left|v_{j, k}\right|\right\}$, denote the global bound on the values of the velocities.

Theorem 4.1. Consider the two-dimensional central scheme (2.5)-(2.6), complemented by the streamfunction computation of the velocity field (3.4)-(3.6). Assume that the discrete slopes, $\omega^{\prime}$ and $\omega^{\prime}$, are reconstructed using the $\theta$-dependent Min-Mod limiter (3.8). Then for any $\theta<2$ there exists a constant, $C_{\theta}=\frac{\sqrt{36+10 \theta(2-\theta)}-6}{20 \theta}$, such that if the CFL condition is fulfilled,

$$
\max (\lambda, \mu) \cdot U_{\infty} \leq C_{\theta}
$$

then the following local maximum principle holds

$$
\min _{\substack{\left|p-\left(j+\frac{1}{2}\right)\right|=\frac{1}{2} \\\left|q-\left(k+\frac{1}{2}\right)\right|=\frac{1}{2}}}\left\{\bar{w}_{p, q}^{n}\right\} \leq \bar{w}_{j+\frac{1}{2}, k+\frac{1}{2}}^{n+1} \leq \max _{\substack{\left|p-\left(j+\frac{1}{2}\right)\right|=\frac{1}{2} \\\left|q-\left(k+\frac{1}{2}\right)\right|=\frac{1}{2}}}\left\{\bar{w}_{p, q}^{n}\right\} .
$$

Remark. Of course, the CFL bound $C_{\theta}$, is far from the optimal $C_{\theta}=\frac{1}{2}$. 
Proof. The main idea is to rewrite $\bar{\omega}_{j+\frac{1}{2}, k+\frac{1}{2}}^{n+1}$ as a convex combination of the cell averages at $t^{n}, \bar{\omega}_{j, k}^{n}, \bar{\omega}_{j+1, k}^{n}, \bar{\omega}_{j, k+1}^{n}, \bar{\omega}_{j+1, k+1}^{n}$. We start by writing $\bar{\omega}_{j+\frac{1}{2}, k+\frac{1}{2}}^{n+1}$ as a sum of five terms

$$
\bar{\omega}_{j+\frac{1}{2}, k+\frac{1}{2}}^{n+1}=\frac{1}{4} \times\left\{\mathcal{I}_{1}+\mathcal{I}_{2}+\mathcal{I}_{3}+\mathcal{I}_{4}+\mathcal{I}_{5}\right\},
$$

with

$$
\begin{aligned}
\mathcal{I}_{1}= & <\bar{\omega}_{., k}^{n}>_{j+\frac{1}{2}}+\frac{\omega_{j, k}^{\prime}-\omega_{j+1, k}^{\prime}}{4}, \mathcal{I}_{2}=<\bar{\omega}_{., k+1}^{n}>_{j+\frac{1}{2}}+\frac{\omega_{j, k+1}^{\prime}-\omega_{j+1, k+1}^{\prime}}{4} \\
\mathcal{I}_{3}= & <\bar{\omega}_{j, .}^{n}>_{k+\frac{1}{2}}+\frac{\omega_{j, k}^{\prime}-\omega_{j, k+1}^{\prime}}{4}, \mathcal{I}_{4}=<\bar{\omega}_{j+1, .}^{n}>_{k+\frac{1}{2}}+\frac{\omega_{j+1, k}^{\prime}-\omega_{j+1, k+1}^{\prime}}{4} \\
\mathcal{I}_{5}= & -2 \lambda\left[\left(f_{j+1, k}^{n+\frac{1}{2}}-f_{j, k}^{n+\frac{1}{2}}\right)+\left(f_{j+1, k+1}^{n+\frac{1}{2}}-f_{j, k+1}^{n+\frac{1}{2}}\right)\right] \\
& -2 \mu\left[\left(g_{j, k+1}^{n+\frac{1}{2}}-g_{j, k}^{n+\frac{1}{2}}\right)+\left(g_{j+1, k+1}^{n+\frac{1}{2}}-g_{j+1, k}^{n+\frac{1}{2}}\right)\right]
\end{aligned}
$$

By the reconstruction of the Min-Mod limiter, $\omega_{j, k}^{\prime}$ and $\omega_{j+1, k}^{\prime}$, cannot have opposite signs (consult [33]), and hence $\mathcal{I}_{1}$ does not exceed

$$
\mathcal{I}_{1} \leq \frac{1}{2}\left(\bar{\omega}_{j, k}^{n}+\bar{\omega}_{j+1, k}^{n}\right)+\frac{\theta}{4}\left|\bar{\omega}_{j+1, k}^{n}-\bar{\omega}_{j, k}^{n}\right| .
$$

Similar bounds hold for $\mathcal{I}_{2}, \mathcal{I}_{3}$ and $\mathcal{I}_{4}$.

Next, we invoke the discrete incompressibility (3.7), which enables us to reformulate $\mathcal{I}_{5}$ as the sum of differences of vorticities

$$
\begin{aligned}
\mathcal{I}_{5}= & -2\left(\lambda u_{j+1, k}^{n+\frac{1}{2}}\right) \cdot\left(\omega_{j+1, k}^{n+\frac{1}{2}}-\omega_{j, k}^{n+\frac{1}{2}}\right) \\
& -2\left(\mu v_{j, k}^{n+\frac{1}{2}}+\lambda u_{j, k}^{n+\frac{1}{2}}-\lambda u_{j+1, k}^{n+\frac{1}{2}}\right) \cdot\left(\omega_{j, k+1}^{n+\frac{1}{2}}-\omega_{j, k}^{n+\frac{1}{2}}\right) \\
& -2\left(\mu v_{j+1, k+1}^{n+\frac{1}{2}}+\lambda u_{j+1, k+1}^{n+\frac{1}{2}}-\mu v_{j+1, k}^{n+\frac{1}{2}}\right) \cdot\left(\omega_{j+1, k+1}^{n+\frac{1}{2}}-\omega_{j, k+1}^{n+\frac{1}{2}}\right) \\
& -2\left(\mu v_{j+1, k}^{n+\frac{1}{2}}\right) \cdot\left(\omega_{j+1, k+1}^{n+\frac{1}{2}}-\omega_{j+1, k}^{n+\frac{1}{2}}\right) .
\end{aligned}
$$

Hence,

$$
\left|\mathcal{I}_{5}\right| \leq 2 U_{\infty}\left[\lambda\left|\mathcal{I}_{51}\right|+(2 \lambda+\mu)\left|\mathcal{I}_{52}\right|+\mu\left|\mathcal{I}_{53}\right|+(\lambda+2 \mu)\left|\mathcal{I}_{54}\right|\right]
$$

with

$$
\begin{aligned}
& \mathcal{I}_{51}:=\omega_{j+1, k}^{n+\frac{1}{2}}-\omega_{j, k}^{n+\frac{1}{2}}, \quad \mathcal{I}_{52}:=\omega_{j, k+1}^{n+\frac{1}{2}}-\omega_{j, k}^{n+\frac{1}{2}}, \\
& \mathcal{I}_{53}:=\omega_{j+1, k+1}^{n+\frac{1}{2}}-\omega_{j, k+1}^{n+\frac{1}{2}}, \quad \mathcal{I}_{54}:=\omega_{j+1, k+1}^{n+\frac{1}{2}}-\omega_{j+1, k}^{n+\frac{1}{2}} .
\end{aligned}
$$

Using the predictor step in its convective form (3.9), the difference between every two neighboring midvalues of the vorticities in each of the $\mathcal{I}_{5 j}, j=1,2,3,4$, 
can be written in terms of the values of the vorticities and the velocity field at time $t=t^{n}$. For example,

$$
\mathcal{I}_{51}=\bar{\omega}_{j+1, k}^{n}-\bar{\omega}_{j, k}^{n}-\frac{\lambda}{2}\left[u_{j+1, k}^{n} \omega_{j+1, k}^{\prime}-u_{j, k}^{n} \omega_{j, k}^{\prime}\right]-\frac{\mu}{2}\left[v_{j+1, k}^{n} \omega_{j+1, k}^{\prime}-v_{j, k}^{n} \omega_{j, k}^{\prime}\right] .
$$

According to the Min-Mod limiter in (3.8), both $\left|\omega_{j+1, k}^{\prime}\right|$ and $\left|\omega_{j, k}^{\prime}\right|$ do not exceed $\theta\left|\bar{\omega}_{j+1, k}^{n}-\bar{\omega}_{j, k}^{n}\right|$; similarly, $\left|\omega_{j+1, k}^{\prime}\right|$ and $\left|\omega_{j, k}^{\prime}\right|$ do not exceed $\theta \mid \bar{\omega}_{j+1, k+1}^{n}-$ $\bar{\omega}_{j+1, k}^{n} \mid$ and $\theta\left|\bar{\omega}_{j, k+1}^{n}-\bar{\omega}_{j, k}^{n}\right|$, respectively. Hence, the term $\mathcal{I}_{51}$ in (4.6) is upperbounded by

$\left|\mathcal{I}_{51}\right| \leq\left(1+\lambda \theta U_{\infty}\right)\left|\omega_{j+1, k}^{n}-\omega_{j, k}^{n}\right|+\frac{\mu}{2} \theta U_{\infty}\left[\left|\omega_{j+1, k+1}^{n}-\omega_{j+1, k}^{n}\right|+\left|\omega_{j, k+1}^{n}-\omega_{j, k}^{n}\right|\right]$.

Similar estimates apply to the remaining terms, $\mathcal{I}_{52}, \mathcal{I}_{53}$ and $\mathcal{I}_{54}$.

Adding all these estimates, we find that $\bar{w}_{j+\frac{1}{2}, k+\frac{1}{2}}^{n+1}$, which we decompose as the sum, $\frac{1}{4} \times\left\{\mathcal{I}_{1}+\mathcal{I}_{51}+\mathcal{I}_{2}+\ldots\right\}$, does not exceed

$$
\begin{aligned}
\frac{1}{4} \times\left\{\frac{1}{2}\left(\bar{\omega}_{j, k}^{n}+\bar{\omega}_{j, k+1}^{n}\right)\right. & \\
& \left.+\left(\frac{\theta}{4}+4 \theta \mu^{2} U_{\infty}^{2}+2(\mu+2 \lambda) U_{\infty}+6 \theta \lambda \mu U_{\infty}^{2}\right)\left|\bar{\omega}_{j, k+1}^{n}-\bar{\omega}_{j, k}^{n}\right|+\ldots\right\}
\end{aligned}
$$

which in turn, does not exceed the maximum of $\left\{\bar{\omega}_{j, k}^{n}, \bar{\omega}_{j+1, k}^{n}, \bar{\omega}_{j, k+1}^{n}, \bar{\omega}_{j+1, k+1}^{n}\right\}$, provided that the following inequalities hold

$$
\begin{aligned}
& \frac{\theta}{4}+4 \theta \mu^{2} U_{\infty}^{2}+2(\mu+2 \lambda) U_{\infty}+6 \theta \lambda \mu U_{\infty}^{2} \leq \frac{1}{2} \\
& \frac{\theta}{4}+4 \theta \lambda^{2} U_{\infty}^{2}+2(2 \mu+\lambda) U_{\infty}+6 \theta \lambda \mu U_{\infty}^{2} \leq \frac{1}{2} .
\end{aligned}
$$

These two inequalities augemented with an analogous treatment for the minimum yield the CFL condition (4.1).

\section{Boundary conditions}

The treatment of boundary conditions in the preset context is of major importance, which is beyond the scope of our paper. Here we assume that such boundary values of the vorticity are given. For the intricate issue of the recovering these vorticity boundary values from the velocity field, we refer to [25], and given these vorticity boundary values, we may then utilize the boundary treatment presented in [23].

In [23], we develop a general staggered non-oscillatory treatment for central schemes in the context of Hyperbolic compressible flows. The main idea is to distinguish between inflow and outflow boundary cells. In inflow cells, we utilize a lower-order reconstruction using the exact point-values given at the boundary; such reconstruction prevents the propagation of spurious oscillations into the interior domain. On outflow boundary cells, however, we extrapolate 
the interior data onto the boundary, and plug these extrapolated values into our central scheme.

An additional critical issue in the current context of incompressible flows, is the treatment of the discrete divergence. In $\S 3$, the velocity reconstruction (3.6) was limited to the periodic framework. Here, we present a more general velocity reconstruction, which is tailored to the non-periodic setup while retaining the discrete incompressibility relation (3.7) required by the maximum principle in $\S 4$. To this end, we define the discrete vorticity at the mid-cells as the average of the four corners of each cell, i.e.

$$
\omega_{j+\frac{1}{2}, k+\frac{1}{2}}:=\frac{1}{4}\left(\omega_{j+1, k+1}+\omega_{j, k+1}+\omega_{j, k}+\omega_{j+1, k}\right) .
$$

We then use a streamfunction, $\psi$, such that $\Delta \psi=-\omega$, which is obtained in these mid-cells, e.g., by solving the five-points Laplacian, $\Delta \psi_{j+\frac{1}{2}, k+\frac{1}{2}}=-\omega_{j+\frac{1}{2}, k+\frac{1}{2}}$. Then, its discrete gradient, $\nabla \psi$ recovers the velocity field, yielding

$$
u_{j, k}=\frac{1}{\Delta y}<\psi_{\cdot, k+\frac{1}{2}}-\psi_{\cdot, k-\frac{1}{2}}>_{j}, \quad v_{j, k}=-\frac{1}{\Delta x}<\psi_{j+\frac{1}{2}, \cdot}-\psi_{j-\frac{1}{2}, \cdot}>_{k} .
$$

Observe that with this integer indexed velocity field, we retain the discrete incompressibility relation (3.7), centered around $\left(j+\frac{1}{2}, k+\frac{1}{2}\right)$, which is required for the consistency between the conservative and convective form - a consistency which is the core of the maximum principle proof in $\S 4$.

\section{Numerical results}

6.1. The "thick" shear-layer problem. Our central scheme was implemented for a two-dimensional model problem taken from [3]. The problem is of a jet in a doubly periodic box, $(0,2 \pi) \times(0,2 \pi)$, governed by the Euler equations (1.2)(1.3). The initial flow consists of a horizontal shear-layer of finite thickness, perturbed by a small amplitude vertical velocity of the form

$$
\begin{aligned}
& u= \begin{cases}\tanh \left(\frac{1}{\rho}(y-\pi / 2)\right) & y \leq \pi \\
\tanh \left(\frac{1}{\rho}(3 \pi / 2-y)\right) & y>\pi\end{cases} \\
& v=\delta \cdot \sin (x) .
\end{aligned}
$$

Here, the "thick" shear-layer width parameter, $\rho$, is taken as $\frac{\pi}{15}$ and the perturbation parameter, $\delta$, equals 0.05 .

The second-order calculations were done with discrete slopes calculated by the "classical" Min-Mod limiter (3.8) with $\theta=1$. The third-order calculations, however, were carried out without limiters (using $\theta \equiv 1$ in (2.9),(2.10)). This is an oscillatory reconstruction, yet remarkably, this does not affect the overall stability and convergence properties of the approximated solution. It is a matter of further investigation to fully understand the reasons for such a behavior.

For this periodic setup, the velocities were reconstructed from the calculated pointvalues of the vorticity using the straightforward spectral method, (3.3), efficiently implemented via the FFT with the complexity of $O\left(n^{2} \log (n)\right)$. 


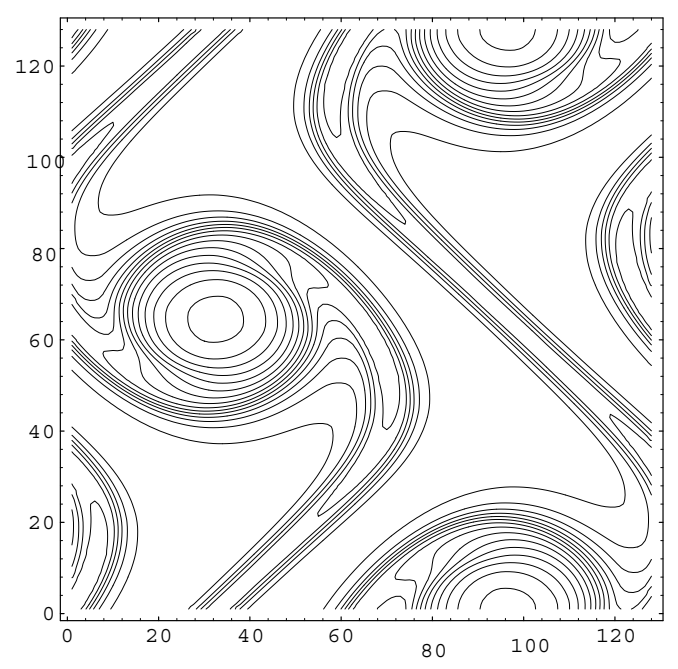

Figure 6.1. "Thick" shear-layer, second-order, t $=8,128 * 128$.

Figure 6.1 displays a typical contour plot of the vorticity. Figures 6.2-6.7 describe the evolution of the vorticity computed using the second-order central scheme (2.5)-(2.6), while figures 6.8-6.13 describe the corresponding results obtained by the third-order central scheme (2.11)-(2.12).

Note that the oscillations in the third-order runs, can be barely noticed. Both, the second and third-order results represent the solution to the desired accuracy; their difference is due to the added high-resolution in the third-order computation. At large times, the second and third-order solution approach each other, due to the embedded dissipation of the schemes (compare Figure 6.7 with Figure 6.12). The lack of sufficient resolution, does not affect the stability of the numerical solution.

Figure 6.20 shows the behavior of the discrete enstrophy in different runs of both the second and the third-order schemes. The origins of all plots were shifted in order to calibrate our comparison of the enstrophy decay. This decay in the enstrophy is due to the embedded numerical viscosity in our scheme (- the Min-Mod limiter decreases the extrema, among other things). Two phenomena can be observed: First, for a fixed time step, $\Delta t$, a finer spatial grid slows down the enstrophy decay rate, which is expected in view of the smaller numerical viscosity. Second, for a fixed spatial grid, a larger time step, $\Delta t$, slows down the enstrophy decay rate, since fewer time steps are taken and hence less numerical dissipation is accumulated. Note that the decay rate in the enstrophy for a $64 * 64$ grid in the third-order scheme, is comparable with the decay in the $128 * 128$ grid for the second-order scheme. Finally, we note that as time evolves, the solution becomes smoother, as smaller under-resolved scales are dissipated. Consequently, the enstrophy decay slows down as evident in Figure 6.20. The behavior of the enstrophy indicates that our central schemes, do supply sufficient resolution at early stages [18]. 


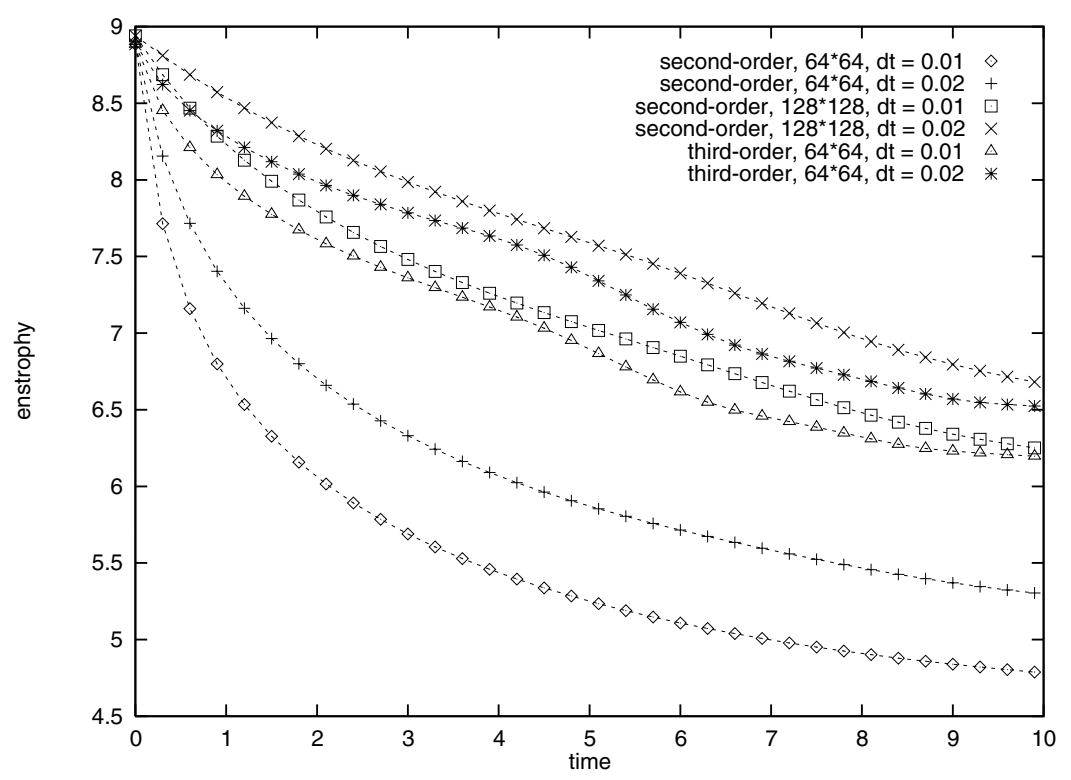

Figure 6.20. Enstrophy plot for the "thick" shear-layer problem.

6.2. The "thin" shear-layer problem. In [4], Brown and Minion revisit the problem of a doubly periodic shear-layer with a "thin" width parameter, $\rho$. They present an upwind Godunov-projection method for the Navier-Stokes equations, and study its behavior as the viscosity term tends to zero. Their results show the appearance of spurious vortices on coarser grids. The beginning of spurious rollups are also evident in some of the calculations of E and Shu [32], who solved the Euler equations at the "thick" shear-layer setup, using an ENO method. Brown and Minion also refer to similar results by Rider and Henshaw, [4], using a Lax-Wendroff method and a centered fourth-order difference primitive variable based method.

Using our scheme, we run several numerical simulations equivalent to those conducted by Brown and Minion. As in the "thick" shear-layer setup, we studied the Euler equations, subject to the initial data (6.1). This time, however, the shear-layer width parameter, $\rho$, was taken as $\frac{\pi}{50}$, and the same $\delta=0.05$ was used.

Figures 6.14-6.19 describe the evolution of vorticity computed by the secondorder central scheme. It can be clearly seen, that there are no spurious vortices in our results. The "thin" shear-layer results show the exact convergence and stability nature of the central scheme, as in the case of a "thick" shear-layer. This again demonstrates the huge potential of our central schemes.

\section{References}

1. C. Anderson, An introduction to vortex methods, Lecture Notes in Math., 1360, SpringerVerlag, Berlin-New York, 1988. 
2. C. Anderson and C. Greengard, On vortex methods, SIAM J. Numer. Anal. , 22 (1985), 413-440.

3. J. B. Bell, P. Colella, and H. M. Glaz, A second-order projection method for the incompressible Navier-Stokes equations, J. Comput. Phys. 85 (1989),257-283.

4. D. L. Brown and M. L. Minion, Performance of under-resolved two-dimensional incompressible flow simulations, J. Comput. Phys. 122 (1995), 165-183.

5. T. Chacon-Rebollo and T. Hou, A Lagrangian finite element method for the 2-D Euler equations, Comm. Pure. Appl. Math. 43 (1990), 735-767.

6. A. J. Chorin, A numerical method for solving incompressible viscous flow problems, J. Comput. Phys. 2 (1967), 12-26.

7. __ Numerical solution of the Navier-Stokes equations, Math. Comp. 22 (1968), 745762.

8. R. DiPerna and A. Majda, Concentrations in regularizations for 2-D incompressible flow, Comm. Pure. Appl. Math. 40 (1987), 301-345.

9. K. O. Friedrichs and P. D. Lax, Systems of conservation equations with a convex extension, Proc. Nat. Acad. Sci. 68 (1971), 1686-1688.

10. E. Godlewski and P.-A. Raviart, Hyperbolic systems of conservation laws, Mathematics and Applications, Ellipses, Paris, 1991.

11. O. H. Hald, Convergence of random methods for a reaction-diffusion equation, SIAM J. Sci. Stat. Comp. 2 (1981), 85-94.

12. Convergence of Fourier methods for Navier-Stokes equations, J. Comput. Phys. 40 (1981), 305-317.

13. A. Harten, High resolution schemes for hyperbolic conservation laws, J. Comput. Phys. 49 (1983), 357-393.

14. T. Y. Hou and B. T. R. Wetton, Second-order convergence of a projection scheme for the incompressible Navier-Stokes equations with boundaries, SIAM J. Numer. Anal. 30 (1993), 609-629.

15. G.-S. Jiang and E. Tadmor, Nonoscillatory central schemes for multidimensional hyperbolic conservation laws, UCLA CAM Report, No. 96-36 (1996).

16. R. Kupferman and E. Tadmor, A fast high-resolution second-order central scheme for incompressible flows, UCLA CAM Report, No. 96-51 (1996).

17. R. Krasny, Computing vortex sheet motion, Proc. Internat. Congress Math., Vol. I, II (Kyoto, 1990), 1573-1583, Math. Soc. Japan, Tokyo, 1991.

18. H.-O. Kreiss, private communication.

19. B. van Leer, Towards the ultimate conservative difference scheme, $V$. A second-order sequel to Godunov's method, J. Comput. Phys. 32 (1979), 101-136.

20. R. J. LeVeque, High-resolution conservative algorithms for advection in incompressible flow, SIAM J. Numer. Anal. 33 (1996), 627-665.

21. __ Numerical methods for conservation laws, Lectures in Math., Birkhauser, Springer-Verlag, Basel, 1992.

22. D. Levy and E. Tadmor, in preparation.

23. Non-oscillatory boundary treatment for staggered central schemes, in preparation.

24. X.-D. Liu, S. Osher, Nonoscillatory high order accurate self-similar maximum principle satisfying shock capturing schemes I, SINUM 33 (1996), 760-779.

25. X.-D. Liu and E. Tadmor, A Non-oscillatory upwind scheme for incompressible NavierStokes flows, preprint.

26. __ Third order nonoscillatory central scheme for hyperbolic conservation laws, Numer. Math. (to appear).

27. J. S. Lowengrub, M. J. Shelley, and B. Merriman, High-order and efficient methods for the vorticity formulation of the Euler equations, SIAM J. Sci. Comp. 14 (1993), 1107-1142.

28. H. Nessyahu and E. Tadmor, Non-oscillatory central differencing for hyperbolic conservation laws, J. Comput. Phys. 87 (1990), 408-463. 
29. S. Osher and E. Tadmor, On the convergence of difference approximations to scalar conservation laws, Math. Comp. 50 (1988), 19-51.

30. P. A. Raviart, An analysis of particle methods, Numerical methods in fluid dynamics (Como, 1983), 243-324, Lecture Notes in Math., 1127, Springer, Berlin-New York, 1985.

31. P. L. Roe, Approximate Riemann solvers, parameter vectors, and difference schemes, J. Comput. Phys. 43 (1981), 357-372.

32. C.-W. Shu and W. E, A numerical resolution study of high order essentially non-oscillatory schemes applied to incompressible flow, J. Comput. Phys. 110 (1994), 39-46.

33. P. K. Sweby, High resolution schemes using flux limiters for hyperbolic conservation laws, SIAM J. Numer. Anal. 21 (1984), 995-1011.

34. L. Tartar, Compensated compactness and applications to partial differential equations, Nonlinear Analysis and Mechanics, Herriot-Watt Symposium, IV, pp. 136-212, Res. Notes in Math., 39, Pitman, Boston, Mass.-London, 1979.

D. L. \& E. T.: School of Mathematical Sciences, Tel-Aviv University, Tel-Aviv 69978, ISRAEL

E. T.: Department of Mathematics, UClA, Los Angeles, CA 90095

E-mail address: tadmor@math.ucla.edu, dlevy@math.tau.ac.il 